\title{
Interference Distribution of a CDMA Cognitive Radio Ad Hoc Network
}

\author{
Miguel Luís ${ }^{1,2}$, Rodolfo Oliveira ${ }^{1}$, Rui Dinis ${ }^{1,2}$, and Luis Bernardo ${ }^{1}$ \\ ${ }^{1}$ CTS, Uninova, Dep. ${ }^{\circ}$ de Eng. ${ }^{a}$ Electrotécnica, Faculdade de Ciências e Tecnologia, FCT, \\ Universidade Nova de Lisboa, 2829-516, Caparica, Portugal \\ ${ }^{2}$ IT, Instituto de Telecomunicações, Portugal \\ nmal@campus.fct.unl.pt, \{rado,rdinis, lflb\}@fct.unl.pt
}

\begin{abstract}
It is well known that in ad hoc networks the interference at a given receiver is expressed by the sum of several random variables representing the distinct sources of interference, and no exact closed-form distribution is known for such a sum. This work characterizes the interference distribution of a Cognitive Radio ad hoc Network (CRAHN) based on Code Division Multiple Access (CDMA). The authors start to explore an analytical model for the multiple-access interference of a Primary User (PU), being thereafter extended to embrace the co-existence of a Secondary User (SU) network. Several scenarios are simulated and the results are compared to the proposed analytical model.
\end{abstract}

Keywords: cognitive radio ad hoc networks, interference, code-division multiple-access.

\section{Introduction}

Due to the emergence of many wireless communication products and services, spectrum scarcity is increasing. Primary-secondary spectrum sharing has the potential to substantially alleviate this problem. In primary-secondary spectrum sharing, Primary Users (PUs), that represent licensed terminals, can transmit when, where and how they wish, limited only by provisions designed to protect other users of spectrum that are adjacent in frequency or geography. On the other hand, Secondary Users (SUs) are prohibited from any transmission that would cause harmful interference to PUs.

Cognitive Radio ad hoc Networks (CRAHNs) are characterized by a decentralized and autonomous behavior of SUs. One of the most important features supporting the cognitive radio is the ability of sensing the radio spectrum: SUs' transmissions cannot cause harmful interference to PUs, and so SUs must inhibit the medium access if there is high probability of causing such effect. But to sense the spectrum and take a decision about the PUs' activity, a priori statistical behavior of PUs can be helpful.

The statistical characterization of PUs' interference has been studied in several works. The work in [1] is, as far as we know, the first one studying the distribution of 
the interference for a Direct-Sequence (DS) CDMA network where the nodes are distributed according to a 2D (two dimension) Poisson point process. Later on, [2] showed that the distribution of the interference in a Poisson field of interferers can be modeled as an $\alpha$-stable distribution, and the result was applied for both DS and frequency hopping (FH) networks. [3] also validated the use of the $\alpha$-stable to model the interference distribution. However it uses a different technique of proof and based on stochastic propagation models. Souryal et al. [4] extended the previous works for a CDMA network with variable modulation, and considered cross-correlation of shadowing from different users. More recently, [5] introduced a mathematical framework for the characterization of network interference in wireless systems for several applications. In this work, we characterize the interference distribution of a CRAHN based on CDMA. We start to explore an analytical model for the multipleaccess interference of the PU network, being thereafter extended to embrace the coexistence of a SU network. In the last part of the paper we present several simulation results to assess the accuracy of the analytical interference distribution.

The rest of this paper is organized as follows. The next section highlights the main contributions of this work. Section 3 presents the system model. Section 4 describes the $\alpha$-stable interference model and its adaption for a CDMA CRAHN. Section 5 validates the proposed interference model and Section 6 summarizes the conclusions.

\section{Contribution to Value Creation}

CRAHNs could enable much more spectrum sharing, thereby unleashing innovative new products and services, provided that we adopt spectrum management policies that are appropriate to the technology. On the other hand, poor choices with respect to technology or policy could fail to achieve the gain in spectral efficiency, or worse, undermine the quality of many existing wireless services.

Not only spectrum sharing is a concern of regulators that need to make more efficient use of spectrum, and substantially alleviate today's debilitating shortage of available spectrum, but also it would be a concern of licensed-holders such as a cellular service. In this scenario, this work addresses the characterization of the interference of a CDMA CRAHN. Our contribution mainly relies in an approximation for the distribution of the interference, which constitutes a useful step to properly devise and parameterize novel schemes for CRAHNs, such as more efficient spectrum sensing techniques.

\section{System Overview}

This work considers a cognitive radio network with $k_{P}$ Primary Users and $k_{S}$ Secondary Users. Both PUs and SUs are randomly distributed in an infinite plane according to a 2D Poisson point process, with an average density of $\lambda_{P}$ and $\lambda_{S}$ nodes per unit area, respectively. The PU receiver is located at the origin.

The system is slotted, and a node transmits a frame with probability $p$ in any given slot. PUs and SUs use CDMA channel access, modeled with random spreading and 
asynchronism at the chip level. In this work we adopt the same symbol duration for both PUs and SUs, and longer chip duration for PUs, i.e. $T_{C P}>T_{C S}$, where $T_{C P}$ and $T_{C S}$ denote the PU's and SU's chip duration, respectively.

The transmitted energy per symbol is assumed to be fixed and the same follows for each set of transmitters (PUs and SUs). The distance between any two nodes is assumed to be greater than the far-field distance of the antenna. The overall channel attenuation for a link is a combination of large-scale path loss, with exponent $m$, and a random propagation attenuation $C$ that can accommodate frequency-flat fading and/or shadowing. The fading/shadowing attenuation is normalized to have unit mean.

\subsection{Primary User Detector Output}

Since this work aims to investigate the multiple-access interference experienced in a licensed user, we only model the received signal and the multiple-access interference at a PU receiver - thereafter represented by the index $j$. A similar analysis can be made for a SU receiver.

The signal received by a PU during a symbol period $0<t<T s$ is given by:

$$
r(t)=\sum_{k=1}^{k_{P}} \sqrt{\frac{C_{P_{k}} E_{T P}}{2 r_{P_{k}}^{m}}}\left[a_{I, k} s_{I, k}(t)+a_{Q, k} s_{Q, k}(t)\right]+\sum_{k=1}^{k_{S}} \sqrt{\frac{C_{S_{k}} E_{T S}}{2 r_{S_{k}}^{m}}}\left[a_{I, k} s_{I, k}(t)+a_{Q, k} s_{Q, k}(t)\right]+n(t)
$$

where the first summation is over all primary transmitting nodes, and the second one is over all the secondary transmitting nodes. $r_{P_{k}}$ (or $r_{S_{k}}$ ) is the distance between the node $k_{P}$ (or $k_{S}$ ) and the receiver; $E_{T P}$ and $E_{T S}$ are proportional to the average transmitted symbol energy; $a_{I, k}$ and $a_{Q, k}$ are the in-phase and quadrature informationbearing amplitudes of node $k_{P}$ and $k_{S}$ (each with unit mean square value), and $n(t)$ is a white Gaussian noise process with two-sided power spectral density $N_{0} / 2$. Signals $s_{I, k}(t)$ and $s_{Q, k}(t)$ are unit energy spreading waveforms defined, as a general form, as:

$$
\begin{aligned}
& s_{I, k}(t)=\sqrt{\frac{2}{T_{S}}} \cos \left(\omega_{c} t+\theta_{k}\right) \sum_{n=0}^{G-1} c_{I, k, n} h\left(t-\tau_{k}-n T_{C}\right) \\
& s_{Q, k}(t)=\sqrt{\frac{2}{T_{S}}} \cos \left(\omega_{c} t+\theta_{k}\right) \sum_{n=0}^{G-1} c_{Q, k, n} h\left(t-\tau_{k}-n T_{C}\right)
\end{aligned}
$$

where $\omega_{C}$ is the carrier angular frequency, $\theta_{k}$ and $\tau_{k}$ are the phase and delay, respectively, of node $k$ 's signals, relative to the receiver. $\left\{c_{I, k, n}\right\}$ and $\left\{c_{Q, k, n}\right\}$ are node $k$ 's in-phase and quadrature (random) spreading sequences, respectively, and are assumed to be made up of independent, equiprobable $\{-1,+1\}$ chips. $h(t)$ is a pulse of duration $T_{C}$ seconds and energy $T_{C}$ Joules. $T_{C}$ becomes $T_{C P}$ or $T_{C S}$ whether we are considering a PU or a SU energy spreading waveform.

Let the two-dimensional vector $\mathbf{U}$ represents the outputs of the in-phase and quadrature detectors matched to the $j$-th signal, sampled at time $t=T_{S}$. Then, 


$$
\boldsymbol{U}=\int_{0}^{T_{S}} r(t) \mathbf{s}_{j}(t) d t=\sqrt{\frac{C_{j} E_{T}}{2 r_{j}^{m}}} \mathbf{a}_{j}+\sqrt{\frac{E_{T}}{2}} \mathbf{Y}+\mathbf{N}
$$

where $\mathbf{s}_{j}(t)=\left[\begin{array}{ll}s_{I, j}(t) & s_{Q, j}(t)\end{array}\right]^{T}$ and $\mathbf{a}_{j}(t)=\left[\begin{array}{ll}a_{I, j}(t) & a_{Q, j}(t)\end{array}\right]^{T}$. The first term in (1) represents the desired signal component and $\mathbf{N}$ is a zero-mean bivariate Gaussian random vector with covariance matrix $\frac{N_{0}}{2} \mathbf{I}$ representing the contribution due to the thermal noise. $\mathbf{Y}$ is the contribution due to the Multiple Access Interference (MAI) caused by PUs and SUs, and is defined as:

$$
\mathbf{Y}=\mathbf{Y}_{P}+\mathbf{Y}_{S}=\sum_{\substack{k=1 \\ k \neq j}}^{k_{P}} \frac{1}{r_{P_{k}}^{m / 2}} \mathbf{X}_{P_{k}}+\sum_{\substack{k=1 \\ k \neq j}}^{k_{S}} \frac{1}{r_{S_{k}}^{m / 2}} \mathbf{X}_{S_{k}}
$$

where $\mathbf{X}_{P}$ and $\mathbf{X}_{S}$ are the components of the overall MAI $\left(\mathbf{X}=\mathbf{X}_{P}+\mathbf{X}_{S}\right)$ due to the primary and secondary interferers, $k_{P}$ and $k_{S}$ respectively, and are expressed as:

$$
\begin{aligned}
& \mathbf{X}_{P}=\left[\begin{array}{ll}
X_{P, I} & X_{P, Q}
\end{array}\right]^{T}=\sqrt{C_{k_{P}}} \mathbf{R}_{P}\left(\Delta_{P_{k} j}, \theta_{P_{k} j}\right) \mathbf{a}_{k_{P}} \\
& \mathbf{X}_{S}=\left[\begin{array}{ll}
X_{S, I} & X_{S, Q}
\end{array}\right]^{T}=\sqrt{C_{k_{S}}} \mathbf{R}_{S}\left(\Delta_{S_{k} j}, \theta_{S_{k} j}\right) \mathbf{a}_{k_{S}} .
\end{aligned}
$$

The general $2 \times 2$ matrix $\mathbf{R}\left(\Delta_{k j}, \theta_{k j}\right)$ is the cross-correlation matrix of nodes $k$ and $j$ 's spreading waveforms. Since we are considering a scenario where there are two distinct chip durations, the cross-correlation matrix $\mathbf{R}$ must be also defined for two distinct scenarios, depending on the origin of the interference, i.e. i) when the interference is caused by PUs (interferers and receiver have the same chip duration $T_{C P}$ ) and ii) when the interference is caused by SUs (interferers and receiver have different chip durations $T_{C P}$ and $T_{C S}$ respectively).

Cross-correlation matrix $\mathbf{R}_{P}$ between two PUs. In this scenario, both interferer and receiver are PUs and the chip duration $T_{C P}$ is the same for both terminals. Then, the cross-correlation matrix $\mathbf{R}_{P}$ is defined as:

$$
\mathbf{R}_{P}\left(\Delta_{P_{k} j}, \theta_{P_{k} j}\right) \triangleq \int_{0}^{T_{S}} \mathbf{s}_{k_{P}}(t) \mathbf{s}_{j}^{T}(t) d t
$$

where $\Delta_{P_{k} j}=\left(\tau_{k_{P}}-\tau_{j}\right) / T_{C P}$ is the normalized relative delay between the signals of nodes $j$ and $k_{P}$, and $\theta_{P_{k} j}=\theta_{k_{P}}-\theta_{j}$ is the corresponding relative phase difference. As an example, the upper-right element of $\mathbf{R}_{\boldsymbol{P}}\left(\Delta_{P_{k} j}, \theta_{P_{k} j}\right)$, which is the correlation between the in-phase spreading sequence of node $k_{P}$ and the quadrature spreading sequence of node $j$, can be expressed as:

$$
\begin{aligned}
\mathbf{R}_{P, I Q}\left(\Delta_{P_{k} j}, \theta_{k_{P} j}\right)= & \int_{0}^{T_{S}} \mathbf{s}_{I, k_{P}}(t) \mathbf{s}_{Q, j}^{T}(t) d t \\
& =-\sin \left(\theta_{P_{k} j}\right)\left[H_{0, P}\left(\Delta_{P_{k} j}\right) \rho_{I Q, P}\left(k_{P}, j ; 0\right)+H_{1, P}\left(\Delta_{P_{k} j}\right) \rho_{I Q, P}\left(k_{P}, j ; 1\right)\right]
\end{aligned}
$$

where:

$$
\rho_{I Q, P}\left(k_{P}, j ; l\right)=\frac{1}{G_{P}} \sum_{n=0}^{G_{P}} c_{I, k_{P}, n} c_{Q, j, n+l}
$$




$$
\begin{gathered}
H_{0, P}\left(\Delta_{P_{k} j}\right)=\int_{\Delta_{P_{k} j}}^{1} h\left(\lambda T_{C P}\right) h\left[\left(\lambda-\Delta_{P_{k} j}\right) T_{C P}\right] d \lambda \\
H_{1, P}\left(\Delta_{P_{k} j}\right)=\int_{0}^{\Delta_{P_{k} j}} h\left(\lambda T_{C P}\right) h\left[\left(\lambda-\Delta_{P_{k} j}+1\right) T_{C P}\right] d \lambda
\end{gathered}
$$

are the cross-correlation of nodes $k_{P}$ and $j$ 's in-phase and quadrature spreading sequences and the partial cross-correlations of the chip pulse, respectively. The phase difference $\theta_{P_{k} j}$ is a uniform random variable in the interval $(0,2 \pi)$, and assuming $0<\tau_{k_{P}}-\tau_{j}<T_{C P}, \Delta_{P_{k} j}$ is uniformly distributed in $(0,1)$. The partial crosscorrelations of the chip pulse, $H_{0, P}$ and $H_{1, P}$, are represented in the Fig. 1.

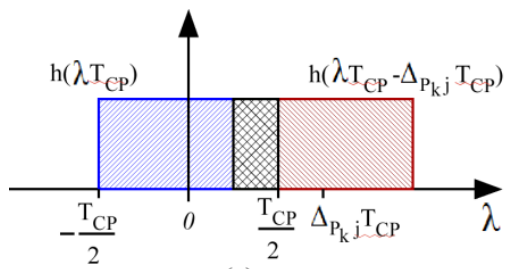

(a)

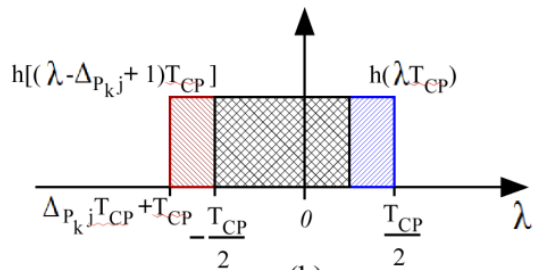

(b)

Fig. 1. Cross-correlations between the PU receiver chip pulse (in blue) and the SU transmitter chip pulse (in red): (a) partial cross-correlation $H_{0, P}$; (b) partial cross-correlation $H_{1, P}$

Cross-correlation matrix $\mathbf{R}_{S}$ between PUs and SUs. In this scenario the receiver is a PU while the interferer belongs to the secondary network. Thus, the chip duration of the involved terminals is not the same. In this work we have assumed an higher chip duration for the PU receiver than for the SU transmitter, i.e. $T_{C P}=\delta T_{C S}$, where $1<\delta<2$.

In this case, the cross-correlation matrix $\mathbf{R}_{S}$ between PUs and SUs can be expressed as

$$
\mathbf{R}_{\boldsymbol{S}}\left(\Delta_{S_{k} j}, \theta_{k_{S} j}\right) \triangleq \int_{0}^{T_{S}} \mathbf{s}_{k_{S}}(t) \mathbf{s}_{j}^{T}(t) d t
$$

where $\Delta_{S_{k} j}=\left(\tau_{k_{S}}-\tau_{j}\right) / T_{C P}$ and $\theta_{S_{k} j}=\theta_{k_{S}}-\theta_{j}$. The phase difference $\theta_{S_{k} j}$ is a uniform random variable in the interval $(0,2 \pi)$, and assuming $0<\tau_{k_{P}}-\tau_{j}<\delta T_{C S}=T_{C P}, \Delta_{S_{k}} j$ is also uniform in $(0,1)$. Fig. 2 presents the cross-correlation between the PU receiver chip pulse and SU transmitter chip pulses when there is no deviation between the SU chip pulse and the PU chip pulse, i.e. $\Delta_{S_{k} j}=0$.

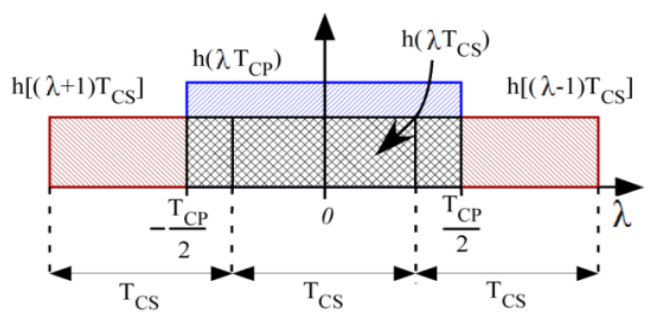

Fig. 2. Cross-correlations between the PU receiver chip pulse (in blue) and three SU transmitter chip pulses (in red) when $\Delta_{S_{k} j}=0$ 
As we did for the previous scenario, the upper-right element of $\mathbf{R}_{\boldsymbol{S}}\left(\Delta_{S_{k} j}, \theta_{S_{k} j}\right)$ can be written as follows

$$
\begin{aligned}
\mathbf{R}_{S, I Q}\left(\Delta_{S_{k} j}, \theta_{S_{k} j}\right)= & \int_{0}^{T_{S}} \mathbf{s}_{I, k_{S}}(t) \mathbf{s}_{Q, j}^{T}(t) d t \\
& =-\sin \left(\theta_{S_{k} j}\right)\left[H_{0, S}\left(\Delta_{S_{k} j}\right) \rho_{I Q, S}\left(k_{S}, j ; 0\right)+H_{1, S}\left(\Delta_{S_{k} j}\right) \rho_{I Q, S}\left(k_{S}, j ; 1\right)\right]
\end{aligned}
$$

where

$$
\begin{gathered}
\rho_{I Q, S}\left(k_{S}, j ; l\right)=\frac{1}{G_{S}} \sum_{n=0}^{G_{S}} c_{I, k_{S}, n} c_{Q, j, n^{\prime}+l} \\
H_{0, S}\left(\Delta_{S_{k} j}\right)=\int_{\Delta_{S_{k} j}}^{1} h\left(\lambda T_{C P}\right) h\left[\lambda T_{C S}-\Delta_{S_{k} j} T_{C P}\right] d \lambda+\int_{\Delta_{S_{k} j}}^{1} h\left(\lambda T_{C P}\right) h\left[(\lambda-1) T_{C S}-\Delta_{S_{k} j} T_{C P}\right] d \lambda \\
+\int_{\Delta_{S_{k} j}}^{1} h\left(\lambda T_{C P}\right) h\left[(\lambda+1) T_{C S}-\Delta_{S_{k} j} T_{C P}\right] d \lambda \\
H_{1, S}\left(\Delta_{S_{k} j}\right)=\int_{0}^{\Delta_{S_{k} j}} h\left(\lambda T_{C P}\right) h\left[\lambda T_{C S}+\Delta_{S_{k} j} T_{C P}\right] d \lambda+\int_{0}^{\Delta_{S_{k} j}} h\left(\lambda T_{C P}\right) h\left[(\lambda-1) T_{C S}+\Delta_{S_{k} j} T_{C P}\right] d \lambda \\
+\int_{0}^{\Delta_{S_{k} j}} h\left(\lambda T_{C P}\right) h\left[(\lambda+1) T_{C S}+\Delta_{S_{k} j} T_{C P}\right] d \lambda
\end{gathered}
$$

and $n^{\prime}=n \operatorname{div} \delta T_{C S}$ is an approximation that takes into account the different lengths of chip durations ${ }^{1}$.

\section{Interference Model}

[2] and [3] modeled the interference of CDMA ad hoc networks using an $\alpha$-stable distribution. Basically, this was achieved by validating the following conditions:

i. $k$ interferers must be distributed in an infinite plane according to a twodimensional Poisson point process with parameter $\lambda_{t}$;

ii. the interference vector $\mathbf{X}_{\mathbf{k}}$ must be i.i.d. (independent and identically distributed) with a spherically symmetric probability density function.

By this way, the authors concluded that the total interference at the detector, $\mathbf{Y}$, is spherically symmetric $\alpha$-stable with characteristic function

$$
\phi_{\boldsymbol{Y}}=\exp \left(-\sigma_{\boldsymbol{Y}}\|\omega\|^{\alpha}\right) \text {. }
$$

The index of stability $\alpha$ and the parameter $\sigma_{Y}$ are given by $\alpha=4 / m$ for $m>2$ and $\sigma_{Y}=-\pi \lambda_{t} \int_{0}^{\infty} \frac{\Phi_{0}^{\prime}(x)}{x^{\alpha}} d x$, respectively, where $\Phi_{0}(\|\omega\|)=\Phi_{X}(\omega)$ is the spherically symmetric characteristic function of $\mathbf{X}$.

An equivalent expression for $\sigma_{\boldsymbol{Y}}$ is given by $\sigma_{\boldsymbol{Y}}=-\pi \lambda_{t} D_{\alpha}^{-1} E\left[|X|^{\alpha}\right][3]$ where:

${ }^{1}$ div denotes the integer division operation on integers. 


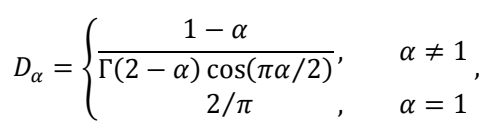

where $\Gamma(\cdot)$ is the gamma function. $X$ represents one component of the spherically symmetric random vector $\mathbf{X}_{\mathbf{k}}$, and $E\left[|X|^{\alpha}\right]$ is given by [4]:

$$
E\left[|X|^{\alpha}\right]=\sqrt{\frac{2^{\alpha}}{\pi G^{\alpha}}} \Gamma\left(\frac{\alpha+1}{2}\right) E\left[C^{\frac{\alpha}{2}}\right] \gamma_{\alpha} \chi_{\alpha}
$$

where the expectation on the right-hand side of Eq. (20) is the $\alpha / 2$-moment of the channel gain. The factor $\gamma_{\alpha}$, related to the shape of the chip pulses, is evaluated using the fact that $\Delta$ is uniformly distributed in $(0,1)$. The factor $\chi_{\alpha}$, concerning the type of the modulation, is likewise evaluated from the uniform distribution of $\theta$ in $(0,2 \pi)$ as well as a probability assignment on the modulation schemes in use.

The objective of this work is to determine if, and how well, this interference model applies to the previous described scenario of a DS/CDMA cognitive radio network. However, before analyzing the application of this model on the previously described cognitive scenario, we will briefly introduce a work developed by Souryal et al. [4] where it is shown that the $\alpha$-stable interference model can be applied in a scenario formed by only PUs. Departing from [4] we seek to employ the same modeling rationale to embrace the co-existence of secondary users.

Brief overview of [4] - $\alpha$-stable interference model for ad hoc CDMA network. To prove that an $\alpha$-stable distribution can be used to model the interference of an $a d$ hoc network using CDMA, Souryal et al. demonstrated that both conditions i) and ii) are met.

The first condition holds because the nodes are positioned according to a Poisson distribution with parameter $\lambda$ and each node transmits independently with probability $p$. Therefore, the distribution of the interferer is also Poisson with parameter $\lambda_{t}=\lambda p$.

The second condition, which requires that $\mathbf{X}_{\mathbf{k}}$ is independent and identical distributed with a spherically symmetric probability density function, is validated in two phases. Firstly, $\mathbf{X}_{\mathbf{k}}$ must be independent and identical distributed. From Eq. (6) the interference vector $\mathbf{X}_{\mathbf{k}}$ becomes $\mathbf{X}_{\boldsymbol{k}}=\mathbf{X}_{\boldsymbol{P}}=f\left(C_{k_{P}}, \Delta_{P_{k} j}, \theta_{P_{k} j}, \mathbf{a}_{k_{P}}\right)$, where $C_{k_{P}}$ is i.i.d. since the propagation conditions are the same for all nodes, i.e., obtained by using identical distributions and independent realizations; $\Delta_{P_{k} j}$ and $\theta_{P_{k} j}$ are uniformly distributed in $(0,1)$ and $(0,2 \pi)$, respectively, with independent realizations; and $\mathbf{a}_{k_{P}}$ is also i.i.d., because all the nodes use the same values of information-bearing amplitudes. Thereby, and regarding the fact that all the arguments of $f(\cdot)$ are mutually independent, $\mathbf{X}_{\mathbf{k}}$ is i.i.d..

Regarding the spherically symmetric probability density function of $\mathbf{X}_{\mathbf{k}}$, this condition is not met in general. In fact, for synchronous CDMA this condition is not met at all. However, departing from [2], Souryal shows that the addition of chip delay and phase asynchronism increases the spherical symmetry. Furthermore, [4] also shows that increasing the spreading factor also improves the spherical symmetry of the density of $\mathbf{X}$. 
Extending [4] to a cognitive radio network. In the next steps we validate the conditions described in [4] in a cognitive radio network scenario.

Regarding the condition i), PUs and SUs transmitters are randomly distributed in an infinite plane according to a $2 \mathrm{D}$ Poisson point process, with an average density of $\lambda_{P}$ and $\lambda_{S}$ (nodes per unit area), respectively. In this case, the overall distribution of nodes positions with intensity $\lambda_{T}=\lambda_{P}+\lambda_{S}$ is also a $2 \mathrm{D}$ Poisson point process.

Let us now validate the second condition. In this scenario we have $\mathbf{X}_{\boldsymbol{k}}=$ $\mathbf{X}_{\boldsymbol{P}}+\mathbf{X}_{S}=f\left(C_{k_{P}}, C_{k_{S}}, \Delta_{P_{k} j}, \Delta_{S_{k} j}, \theta_{P_{k} j}, \theta_{S_{k} j}, \mathrm{a}_{k_{P}}, \mathrm{a}_{k_{S}}\right)$ where $C_{k_{P}}$ and $C_{k_{S}}$ are both i.i.d. since the propagation delay is the same for PUs and SUs; $\Delta_{P_{k} j}$ and $\Delta_{S_{k} j}$ are independent and uniformly distributed in $(0,1) ; \theta_{P_{k} j}$ and $\theta_{S_{k} j}$ are also independent and uniformly distributed in $(0,2 \pi)$; finally $\mathbf{a}_{k_{P}}$ and $\mathbf{a}_{k_{S}}$ are i.i.d. if PUs and SUs use the same values of information-bearing amplitudes, which is the case. Then, and regarding the fact that all the arguments in $f(\cdot)$ are mutually independent, we can also conclude that $\mathbf{X}_{\mathbf{k}}$ is i.i.d..

Regarding the spherical symmetry of $\mathbf{X}_{k}$, the authors have decided to evaluate its validity graphically by plotting the correlation of the spreading waveforms, $\mathbf{R}_{S}$, of two different asynchronous sets of nodes representing the PUs and the SUs, and assuming $G_{P}=11$ and $G_{S}=13$. Observing Fig. 3 we can see that $\mathbf{R}_{S}$ is very close to the unit circle, which means that $\mathbf{X}_{S}$ roughly exhibits a spherically symmetric density, and it can be used as a good approximation [2].

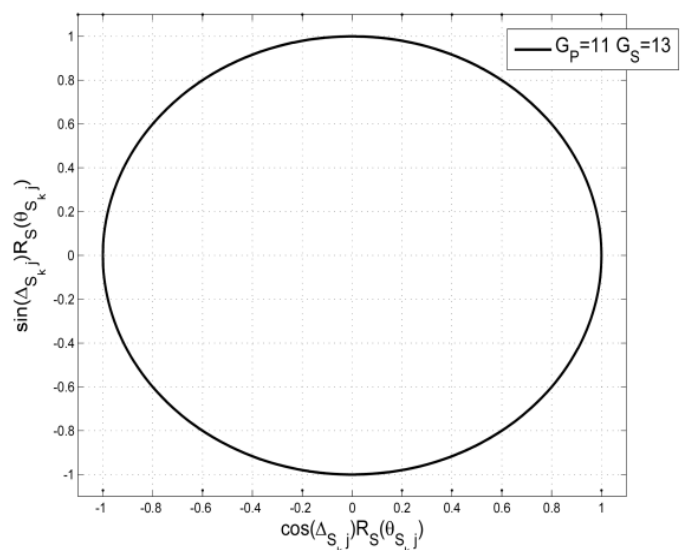

Fig. 3. Spherical symmetry of $\mathbf{R}_{S}$ in a cognitive scenario with $G_{P}=11$ and $G_{S}=13$

\section{$5 \quad$ Model Validation}

To evaluate the validity of the proposed interference model with the considered CRAHN, we compare the distribution of the Interference Energy (IE) given by the model with the one given by the system described in Section 3.

Starting with the model, and following the same rationale described in [4], we can use the fact that a symmetric stable random vector is sub-Gaussian with an underlying Gaussian vector having i.i.d. zero-mean Gaussian components. Then, the $\alpha$-stable 
random vector $\mathbf{Y}$ can be expressed as the product $\mathbf{Y}=\sqrt{V} \cdot \mathbf{T}$, where $\mathbf{T}$ is a zero-mean Gaussian vector with identity covariance matrix, and $V$ is an $\alpha / 2$-stable random vector totally skewed to the right and independent of $\mathbf{T}$ [6]. The scale parameter of $V$ in terms of $\sigma_{\boldsymbol{Y}}$ is

$$
\sigma_{V}=2\left[\sigma_{Y} \cos \left(\frac{\pi \alpha}{4}\right)\right]^{\frac{2}{\alpha}}
$$

According to this, the MAI can be viewed as Gaussian with the random variance, or interference energy $V$, accounting for the randomness of the distances, phases, and chip delays of the interferers. If we consider a scenario with a fourth-power path loss distance $m=4$, the index of stability becomes $\alpha=1$, and the $\alpha / 2$-stable random variable $V$ becomes $1 / 2$-stable with parameter $\sigma_{V}=\sigma_{Y}^{2}$, and with density and distribution functions

$$
\begin{gathered}
f_{V}(v)=\frac{\sigma_{Y}}{\sqrt{2 \pi}} v^{-\frac{3}{2}} \exp \left(-\frac{\sigma_{Y}^{2}}{2 v}\right), \quad v>0 \\
F_{V}(v)=\operatorname{erfc}\left(\frac{\sigma_{Y}}{\sqrt{2 v}}\right), \quad v>0 .
\end{gathered}
$$

On the other hand, the interference energy given by the system is obtained by $I E=10 \log _{10}|\mathbf{Y}|$, where $\mathbf{Y}$ is the MAI given by Eq. (5).

The analytical results for the distribution of the interference energy are compared with simulation results where a PU receiver is located at the origin of a plane with $k=k_{P}+k_{S}$ 2D Poisson distributed interferers with intensity $\lambda_{P}+\lambda_{S}$. Each interferer signal uses quaternary phase-shift keying (QPSK) modulation and rectangular chips. The channel is characterized by being flat fading with a path loss exponent of $m=4$.

Two different scenarios were simulated. In the first one we adopted $k_{P}=200$ PUs and $k_{S}=50$ SUs $(\lambda=2.5$ nodes per unit area), while in the second one the node's density was increased to $k_{P}=200$ PUs and $k_{S}=100$ SUs $(\lambda=3)$. The spreading factors used by each interferer were the same as before, i.e. $G_{P}=11$ and $G_{S}=13$.

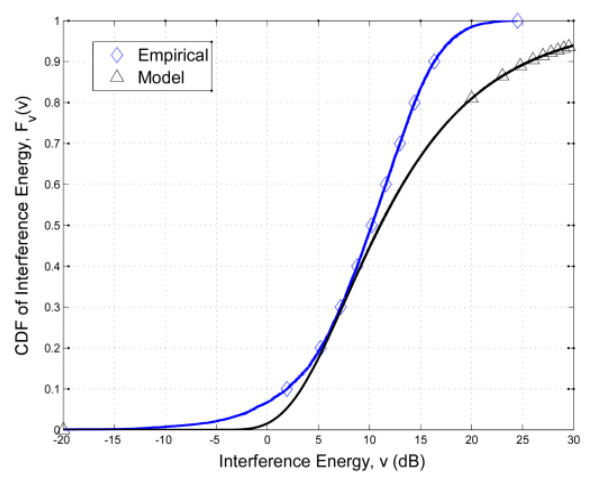

(a)

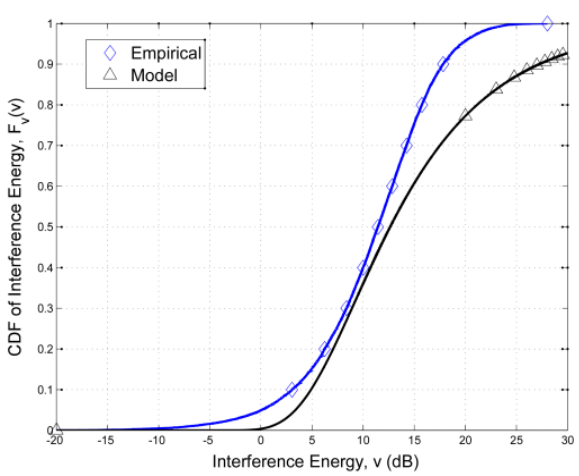

(b)

Fig. 4. CDF of the interference energy: comparison of analytical and empirical results for (a) $\lambda=2.5$ and (b) $\lambda=3$ 
Fig. 4 illustrates the analytical model and empirical results for both scenarios. In both cases, the model captures the trend observed in the simulations (Empirical curve). However, it is noted that for higher values of interference energy, there is a higher deviation between theoretical predictions and the experimental results.

Comparing the empirical interference distribution for both scenarios, we observe that the interference is higher when the system has more interferers, $\lambda=3$. However, this difference $(\simeq 2.5 d B)$ could be even higher if we had considered lower spreading factors for the different set of interferers. This is also one of the reasons that motivated this work. By using our approach, it is now possible to study and characterize the amount of interference caused by the SUs in a primary CDMA CRAHN, and then parameterize the secondary network in order to keep the interference level below a certain threshold that does not harms PUs' communications.

\section{Conclusions}

In this work we have characterized the interference distribution of a CRAHN based on CDMA as an $\alpha$-stable distribution. Starting by exploring an analytical model for the MAI of a single PU network, we have extended it to embrace the co-existence of a SU network. The accuracy of the analytical model for the interference distribution was assessed through several simulations.

For future research, we plan to evaluate the proposed model for different spreading factors and propose a new medium access scheme for SUs mainly based on the insights achieved with this work.

Acknowledgments. This work was partially supported by the European Science Foundation COST Action IC0902 and the Portuguese Science and Technology Foundation under the grant SFRH/BD/68367/2010 and the projects PTDC/EEATEL/115981/2009, PTDC/EEA-TEL/099074/2008, PEst-OE/EEI/LA0008/2011 and PEst-OE/EEI/UI0066/2011.

\section{References}

1. Sousa, E.S., Silvester, J.A.: Optimum transmission ranges in a direct-sequence spreadspectrum multihop packet radio network. IEEE J. Sel. Areas Commu. 8(5), 762-771 (1990)

2. Sousa, S.: Performance of a spread spectrum packet radio network link in a Poisson Point field of interferers. IEEE Trans. Inf. Theory 38(6), 1743-1754 (1992)

3. Ilow, J., Hatzinakos, D., Venetsanopoulos, A.N.: Performance of FH SS radio networks with interference modeled as a mixture of Gaussian and alpha-stable noise. IEEE Trans. Commun. 46(4), 509-520 (1998)

4. Souryal, M.R., Vojcic, B.R., Pickholtz, R.L.: Interference model for ad hoc DS/CDMA packet radio networks with variable coherent modulation. In: Proc. of IEEE Military Communications Conference, vol. 1, pp. 1-6 (October 2003)

5. Win, M.Z., Pinto, P.C., Shepp, L.A.: A Mathematical Theory of Network Interference and Its Applications. Proc. of IEEE 97(2), 205-230 (2009)

6. Samorodnitsky, G., Taqqu, M.S.: Stable Non-Gaussian Random Processes: Stochastic Models with Infinite Variance. Chapman \& Hall, New York (1994) 